\title{
A Fully Discrete Discontinuous Galerkin Method for Nonlinear Fractional Fokker-Planck Equation
}

\author{
Yunying Zheng, ${ }^{1,2}$ Changpin Li, ${ }^{1}$ and Zhengang Zhao ${ }^{1}$ \\ ${ }^{1}$ Department of Mathematics, Shanghai University, Shanghai 200444, China \\ ${ }^{2}$ Department of Mathematics, Huainan Normal University, Huainan 232038, China
}

Correspondence should be addressed to Changpin Li, lcp@shu.edu.cn

Received 13 July 2010; Revised 16 September 2010; Accepted 19 October 2010

Academic Editor: J. Jiang

Copyright (c) 2010 Yunying Zheng et al. This is an open access article distributed under the Creative Commons Attribution License, which permits unrestricted use, distribution, and reproduction in any medium, provided the original work is properly cited.

The fractional Fokker-Planck equation is often used to characterize anomalous diffusion. In this paper, a fully discrete approximation for the nonlinear spatial fractional Fokker-Planck equation is given, where the discontinuous Galerkin finite element approach is utilized in time domain and the Galerkin finite element approach is utilized in spatial domain. The priori error estimate is derived in detail. Numerical examples are presented which are inline with the theoretical convergence rate.

\section{Introduction}

Many models in physics, chemistry are successfully described by the Langevin equation, which has been introduced almost 100 years before. And for some particular cases, say diffusion, the original Langevin equation can be transformed into the Fokker-Planck equation. Hänggi and Thomas [1] associated a Gaussian distribution of the increments of the noise generating process with the classical Fokker-Planck equation. Sun et al. [2] discussed the fractional model for anomalous diffusion. Metzler et al. [3] and Dubkov and Spagnolo [4] derived the fractional Fokker-Planck equation from different anomalous diffusion procedures. Metzler and Klafter [5] discussed fractional kinetic equation and its relation to the fractional Fokker-Planck equation. Dubkov et al. [6] introduced Fokker-Planck equation for Lévy flights. Now the Fokker-Planck equation is one of the best tools for characterizing anomalous diffusion, especially sub-/super-diffusion. Meanwhile the fractional FokkerPlanck equation has been found to be used in relatively wide field of applied sciences, such as plasma physics, population dynamics, biophysics, engineering, neuroscience, nonlinear hydrodynamics, and marking; see [7-13].

The Fokker-Planck equation describes the changes of a random function in space and in time. So different assumptions on probability density function lead to a variety of 
space-time equations. In this paper, we mainly study the model described by the following fractional Fokker-Planck equation, which is a special case in [12]:

$$
\frac{\partial u}{\partial t}=\left({ }_{c} D_{0, x}^{\beta} b(x, t, u)+{ }_{c} D_{0, x}^{2 \beta} a(x, t, u)\right) u, \quad 0<\beta<1,(x, t) \in \Omega \times(0, T],
$$

where ${ }_{C} D_{0, x}^{\beta}$ denotes left fractional derivative with order $\beta$ in the sense of Caputo.

There are some numerical methods to find the approximate solutions of the fractional differential equations [14-21]. But the discontinuous Galerkin finite element method is a very attractive method for partial differential equations because of its flexibility and efficiency in terms of mesh and shape functions. And the higher order of convergence can be achieved without over many iterations. Such a method was first proposed and analyzed in the early 1970s as a technique to seek numerical solutions of partial differential equations. The discontinuous Galerkin finite element method becomes a very attractive tool for the initial problems of the ODEs and the initial-boundary problems of PDEs; see [22-27].

The rest of this paper is constructed as follows. The fractional derivative space is introduced in Section 2. In Section 3, the discontinuous Galerkin finite element scheme is introduced. The existence and uniqueness of numerical solution are proved in Section 3. And the error estimate of the discontinuous Galerkin finite element approximation is studied in Section 4. Finally in Section 5, numerical examples are also taken to show the efficiency of the theoretical results.

\section{Fractional Derivative Space}

In this section, we firstly introduce the fractional integral, fractional Caputo derivative, and their properties.

Definition 2.1. The $\alpha$ th order left and right Riemann-Liouville integrals of function $u(x)$ are defined as follows:

$$
\begin{aligned}
& D_{a, x}^{-\alpha} u(x)=\frac{1}{\Gamma(\alpha)} \int_{a}^{x} \frac{u(s)}{(x-s)^{1-\alpha}} d s, \\
& D_{x, b}^{-\alpha} u(x)=\frac{1}{\Gamma(\alpha)} \int_{x}^{b} \frac{u(s)}{(s-x)^{1-\alpha}} d s,
\end{aligned}
$$

where $\alpha>0$ and $a<x<b$.

Definition 2.2. The $\alpha$ th order left and right Caputo derivatives of function $u(x)$ are defined in

$$
\begin{gathered}
c D_{a, x}^{\alpha} u(x)=D_{a, x}^{\alpha-n} D^{n} u(x), \\
c D_{x, b}^{\alpha} u(x)=D_{x, b}^{\alpha-n}(-D)^{n} u(x),
\end{gathered}
$$

where $n-1<\alpha<n \in Z^{+}$and $a<x<b$. 
Definition 2.3. Let $\alpha>0$; define a fractional derivative space $J_{s}^{\alpha, p}(\Omega)(\Omega=(a, b))$ as

$$
J_{s}^{\alpha, p}(\Omega)=\left\{u(x) \in H^{n}(\Omega):{ }_{C} D_{a, x}^{\alpha} u(x),{ }_{c} D_{x, b}^{\alpha} u(x) \in L^{p}(\Omega), n-1 \leq \alpha<n\right\},
$$

endowed with the seminorm

$$
|u|_{J_{s}^{\alpha, p}}=\left|\left(c D_{a, x}^{\alpha} u(x), c D_{x, b}^{\alpha} u(x)\right)^{1 / 2}\right|_{L^{p}(\Omega)}
$$

and the norm

$$
\|u(x)\|_{J_{s}^{\alpha, p}}=\left(\sum_{k \leq[\alpha]}\left\|D^{\beta} u(x)\right\|^{p}+|u(x)|_{J_{s}^{\alpha, p}}^{p}\right)^{1 / p}, \quad \beta \in Z, 0 \leq \beta \leq[\alpha]-1 .
$$

With the help of Fourier Transform, we can conclude that the following three expressions: $\int_{\Omega}\left|c D_{a, x}^{\alpha} u(x) \cdot{ }_{C} D_{x, b}^{\alpha} u(x)\right| d \Omega, \int_{\Omega}\left({ }_{c} D_{a, x}^{\alpha} u(x)\right)^{2} d \Omega$, and $\int_{\Omega}\left({ }_{c} D_{x, b}^{\alpha} u(x)\right)^{2} d \Omega$, are equivalent [20]. So $\left(\int_{\Omega}\left(c D_{a, x}^{\alpha} u(x)\right)^{2} d \Omega\right)^{1 / 2}$ and $\left(\int_{\Omega}\left(c D_{x, b}^{\alpha} u(x)\right)^{2} d \Omega\right)^{1 / 2}$ can also be recognized as the seminorms of the fractional space $J_{s}^{\alpha, p}$, and when we use the seminorm of fractional space $J_{s}^{\alpha, p}$, there is no difference among them. Now we restrict our discussion in case $p=2$, and the following notations are used, $J_{s}^{\alpha, 2}(\Omega)$ being rewritten as $J_{s}^{\alpha}(\Omega)$ with norm $\|\cdot\|_{J_{s}^{\alpha}}$ or $\|\cdot\|_{\alpha}$ and seminorm $|\cdot|_{J_{s}^{\alpha}}$ or $|\cdot|_{\alpha}$. We denote $J_{s, 0}^{\alpha}(\Omega)$ as the closure of $C_{0}^{\infty}(\Omega)$ under its norm.

The following lemmas are useful for our discussions later on.

Lemma 2.4 (see [19]). Let $n-1 \leq \alpha<n$; if $u \in J_{s, 0}^{\alpha}([a, b])$, then

$$
\begin{aligned}
& \left(D_{a, x}^{-\alpha} C D_{a, x}^{\alpha} u\right)(x)=u(x), \\
& \left(D_{x, b}^{-\alpha} C D_{x, b}^{\alpha} u\right)(x)=u(x) .
\end{aligned}
$$

Lemma 2.5 (see $[20])$. For $u \in J_{s, 0}^{\alpha}([a, b]), 0<\beta<\alpha$, then

$$
{ }_{C} D_{a, x}^{\alpha} u(x)={ }_{C} D_{a, x}^{\alpha-\beta} c D_{a, x}^{\beta} u(x) .
$$

Lemma 2.6. For $u \in C_{0}^{2 \alpha}([a, b]), v \in C_{0}^{\alpha}([a, b]), n-1 \leq \alpha<n$, then

$$
\left(c D_{a, x}^{2 \alpha} u, v\right)=(-1)^{n}\left(c D_{a, x}^{\alpha} u, c D_{x, b}^{\alpha} v\right)
$$

Lemma 2.7 (see [21]). For $u \in J_{s, 0}^{\alpha}(\Omega), 0<\beta<\alpha$, then

$$
|u|_{\beta} \leq|u|_{\alpha}, \quad\|u\|_{\beta} \leq\|u\|_{\alpha} .
$$


For $u \in H_{0}^{\alpha}(\Omega)$, then

$$
\|u\|_{L^{2}(\Omega)} \leq c|u|_{H_{0}^{\alpha}}
$$

For $0<\beta<\alpha$, then

$$
\|u\|_{H_{0}^{\beta}(\Omega)} \leq C|u|_{H_{0}^{\alpha}} .
$$

Lemma 2.8. Let $\mu>0$. The following mapping properties hold:

$$
\begin{aligned}
& { }_{C} D_{a, x}^{\alpha}: J_{s}^{\mu}(\Omega) \longrightarrow L^{2}(\Omega) \text { is a bounded linear operator, } \\
& { }_{C} D_{x, b}^{\alpha}: J_{s}^{\mu}(\Omega) \longrightarrow L^{2}(\Omega) \text { is a bounded linear operator. }
\end{aligned}
$$

The proof is similar to that in [19].

\section{The Space-Time Discontinuous Galerkin Finite Element Approximation}

In this section, we formulate a fully discrete discontinuous Galerkin finite element method for a type of nonlinear spacial fractional Fokker-Planck equation.

Problem 1 (nonlinear fractional Fokker-Planck equation). For $0<\beta<1$,

$$
\begin{gathered}
u_{t}=\left[-{ }_{c} D_{0, x}^{\beta} b(x, t, u)+{ }_{c} D_{0, x}^{2 \beta} a(x, t, u)\right] u, \quad(x, t) \in \Omega \times(0, T], \\
u(x, t)=\phi(x, t), \quad x \in \partial \Omega \times(0, T] \\
u(x, 0)=g(x), \quad x \in \bar{\Omega}
\end{gathered}
$$

where the $\Omega$ is a bounded domain. For positive constants $m$ and $M$, the coefficients $a$ and $b$ satisfy

$$
0<m<a(u)<M, \quad 0<m<b(u)<M .
$$

Throughout the paper, we always assume that the following mild Lipschitz continuity conditions on $a, b$ are satisfied: there exists a positive constant $L$ such that for $x \in \Omega, t \in(0, T]$, and $s, r \in R$, there have

$$
\begin{aligned}
& |a(x, t, s)-a(x, t, r)| \leq L|s-r| \\
& |b(x, t, s)-b(x, t, r)| \leq L|s-r| .
\end{aligned}
$$


Let $\mathcal{K}_{h}=\{K\}$ be a partition of spatial domain $\Omega$. Define $h_{k}$ as the diameter of the element $K$ and $h=\max _{K \in \mathcal{K}_{h}} h_{K}$. And let $S_{h}$ be a finite element space

$$
S_{h}=\left\{v \in H_{0}^{\beta}(\Omega):\left.v\right|_{K} \in P_{r-1}(K), K \in \mathcal{K}_{h}\right\},
$$

where $P_{r-1}(K)$ is a set of polynomials with degree $r-1$ on a given domain $K$. And the functions in $S_{h}$ are continuous on $\Omega$.

Let us consider a partition of time domain $I=(0, T], 0=t_{0}<t_{1}<\cdots<t_{N}=T$, and $I_{n}=\left(t_{n}, t_{n+1}\right], k_{n}=t_{n+1}-t_{n}, n=0,1, \ldots N-1, k=\max k_{n}$. On each time slab $I_{n}$, we define a discrete function space $\mathfrak{I}_{k}$ as

$$
\Im_{k}=\left\{v: I_{n} \longrightarrow R ;\left.v\right|_{t \in I_{n}} \in P_{q}\left(I_{n}\right)\right\}
$$

The functions in the space $\Im_{k}$ can be discontinuous at the time node $t_{n}$, but is at least left continuous and right continuous. And the functions in the space $\mathfrak{I}_{k}$ are polynomials, whose degree is no more than $q-1$.

Define a discrete function space $T_{k h}$ on $\Omega \times I$ as

$$
T_{k h}=\left\{v: \Omega \times I \longrightarrow R \mid \forall t \in I, v(\cdot, t) \in \mathfrak{I}_{k}, \forall x \in \Omega, v(x, \cdot) \in S_{h}\right\}
$$

Moreover, the space $T_{k h}$ can be verified as follows:

$$
T_{k h}=\left\{v \mid v(x, t)=\sum_{j=0}^{q-1} t^{j} x_{j}(x), x_{j} \in S_{h}\right\}
$$

where $q$ is a positive integer. In other words, for each $t \in I_{n}$ the functions in $T_{k h}$ are the elements of $S_{h}$, and for each $x \in \Omega$ piecewise polynomial functions in $t$ of degree $q-1$ with possible discontinuities at the nodes $t_{n}, n=0,1, \ldots, N-1$. Set $T_{k h}^{n}=\left\{\left.v\right|_{\Omega \times I_{n}}: v \in T_{k h}\right\}$.

We introduce some norms on different spaces which will be used later on. And $L^{2}\left(I, J_{s}^{\beta}(\Omega)\right)$ is equipped with the norms

$$
\|v\|_{0, \beta}=\left(\int_{I}\|v\|_{\beta}^{2} d t\right)^{1 / 2}, \quad\|v\|_{\infty, \beta}=\max _{I}\|v\|_{\beta}=\max _{0 \leq t \leq t_{N}}\|v\|_{\beta}
$$

And $L^{2}\left(I_{n}, J_{s}^{\beta}(\Omega)\right)$ is equipped with the norms

$$
\|v\|_{0, \beta, n}=\left(\int_{I_{n}}\|v\|_{\beta}^{2} d t\right)^{1 / 2}, \quad\|v\|_{\infty, \beta, n}=\max _{I_{n}}\|v\|_{\beta}=\max _{t_{n}<t \leq t_{n+1}}\|v\|_{\beta} .
$$


In order to derive a variational form of Problem 1, we assume that $u$ is a sufficiently smooth solution of Problem 1, then multiply an arbitrary $v \in T_{k h}$ to obtain the integration formulation

$$
\int_{I}\left(\frac{\partial u}{\partial t}, v\right) d t=\int_{I}\left(c D_{0, x}^{2 \beta} a(u) u, v\right) d t-\int_{I}\left({ }_{c} D_{0, x}^{\beta} b(u) u, v\right) d t
$$

where $(\cdot, \cdot)$ denotes the inner product on $L^{2}(\Omega)$. Integrating by parts in the right, noting that for all $t \in I, v \in J_{0, s}^{\beta}(\Omega)$, and the discontinuous property at the time node $t_{n}, n=0, \ldots, N-1$, one has

$$
\sum_{n=0}^{N-1}\left\{\int_{I_{n}}\left(\frac{\partial u}{\partial t}+{ }_{c} D_{0, x}^{\beta} b(u) u, v\right)+\left({ }_{c} D_{0, x}^{\beta} a(u) u, c D_{x, b}^{\beta} v\right) d t+\left([u]_{n}, v_{n}^{+}\right)\right\}=0 .
$$

The notation $[u]_{n}$ denotes the jump of the function $u$ at the time node $t_{n}$; that is,

$$
[u]_{n}=\lim _{\varepsilon \rightarrow 0}\left[u\left(\cdot, t_{n}+|\varepsilon|\right)-u\left(\cdot, t_{n}-|\varepsilon|\right)\right] .
$$

Using the superscripts "-" and "+" for left and right limits, respectively, the jump is described by

$$
[u]_{n}=u\left(\cdot, t_{n}^{+}\right)-u\left(\cdot, t_{n}^{-}\right)
$$

It shows the discontinuity of the scheme. The inner product $\left([u]_{n}, v_{n}^{+}\right)$also denotes the transport process during different time-space slabs.

Thus, we define

$$
A(u, v)=\int_{I_{n}}\left\{\left(\frac{\partial u}{\partial t}, v\right)+\left({ }_{c} D_{0, x}^{\beta} a(u) u, c_{C} D_{x, b}^{\beta} v\right)+\left({ }_{c} D_{0, x}^{\beta} b(u) u, v\right)\right\} d t+\left([u]_{n}, v_{n}^{+}\right) .
$$

Definition 3.1. For all $v \in T_{k h}$, the function $u \in T_{k h}$ is a variational solution of Problem 1 provided that

$$
\sum_{n=0}^{N-1} A(u, v)=0, \quad \forall v \in T_{k h}
$$

Now we are ready to describe a fully discrete space-time finite element method to solve the nonlinear problem 1, where the Galerkin finite element method is used in spacial domain and discontinuous finite element approximation is used in time domain.

Applying the same notations with Definition 3.1, the space-time discontinuous Galerkin scheme for Problem 1 can be now formulated as follows. Find $U \in T_{k h^{\prime}}^{n}$, satisfying, for all $v \in T_{k h^{\prime}}^{n} n=0,1, \ldots, N-1$,

$$
A(U, v)=0, \quad \forall v \in T_{k h} .
$$


The value of $U$ on $t_{0}^{-}$is replaced by the initial condition $g(x)$. The term including $U_{0}$ can be moved to the right-hand side of (3.16). Once it is computed, the value of $U$ on $t_{0}^{+}$is different from that on $u_{0}^{-}$, and it is the error introduced by the discretization in the numerical scheme.

Noting the discontinuity at each node $t_{n}, n=0,1, \ldots, N-1$ in $T_{h k}$, the computation of $u_{h}$ can be decoupled in each time slab. Once $u_{h}$ is known on $t_{n}^{-}$, this value is taken as an initial condition for the time slab $I_{n}$ and the following equation needs to be solved:

$$
\left(U_{n+1}^{-}, v_{n+1}^{-}\right)+\int_{I_{n}}\left\{-\left(U, \frac{\partial v}{\partial t}\right)+\left({ }_{c} D_{0, x}^{\beta} a(U) U, c D_{x, b}^{\beta} v\right)+\left({ }_{c} D_{0, x}^{\beta} b(U) U, v\right)\right\} d t=\left(U_{n}^{-}, v_{n}^{+}\right)
$$

Next, we investigate the uniqueness and existence about the numerical scheme. First, we give the scheme in detail. For a fixed integer $q \geq 1$, let $l_{i}(\tau)_{i=1}^{q}$ be the Lagrange polynomials associated with the abscissa $0<\tau_{1}<\cdots<\tau_{q}=1$; that is,

$$
l_{i}(\tau)=\prod_{\substack{j=1, \ldots, q \\ i \neq j}} \frac{\tau-\tau_{j}}{\tau_{i}-\tau_{j}}
$$

For the quadrature in time slab $\left(t_{n}, t_{n+1}\right]$, we use the Radau quadrature rule. For a given function $g(\tau), \tau \in[0,1]$, the following approximation holds:

$$
\int_{0}^{1} g(\tau) d \tau \approx \sum_{j=1}^{q} w_{j} g\left(\tau_{j}\right)
$$

where $w_{j}=\int_{0}^{1} l_{j}(\tau) d \tau$. This quadrature rule is exact for all polynomials of degree $\leq 2 q-2$. Using the linear transformation $t=t_{n}+\tau k_{n}$, which maps $[0,1]$ into $\bar{I}_{n}$, one gets

$$
\begin{gathered}
t_{n, j}=t_{n}+\tau_{j} k_{n}, \quad j=1, \ldots, q, t_{n, q}=t_{n+1}, \\
l_{n, j}(t)=l_{j}(\tau), \quad t=t_{n}+\tau k_{n}, \\
w_{n, j}=\int_{t_{n}}^{t_{n+1}} l_{n, j} d t=k_{n} \int_{0}^{1} l_{j}(\tau) d \tau=k_{n} w_{j}, \quad j=1, \ldots, q .
\end{gathered}
$$

Then the Radau rule in $\bar{I}_{n}$ can be got by

$$
\int_{I_{n}} g(t) d t=\sum_{j=1}^{q} \int_{0}^{1} l_{j}(\tau) d \tau g\left(t_{n}+\tau_{j} k_{n}\right)=\sum_{j=1}^{q} w_{n, j} g\left(t_{n, j}\right) .
$$


We choose $\left\{l_{n, i}(t)\right\}_{j=1}^{q}$ as the basis functions for the piecewise polynomial function space $P_{q-1}\left(I_{n}\right)$. Then $\left.U\right|_{I_{n}}$ is uniquely determined by the functions $U_{n, j}=U_{n, j}(x), U(x) \in S_{h}$, such that

$$
U(x, t)=\sum_{j=1}^{q} l_{n, j}(t) U_{n, j}(x), \quad(x, t) \in \Omega \times I_{n} .
$$

Taking $v=l_{n, i}(t) \psi(x) \in V_{h \mathrm{k}}^{n}$ into (3.17), where $\psi \in S_{h^{\prime}}^{n}$ we have

$$
\begin{aligned}
\left(l_{n, q}\left(t_{n, q}\right) U_{n, q}, l_{n, i}\left(t_{n, q}\right) \psi(x)\right)-\int_{I_{n}}\left(\sum_{j=1}^{q} l_{n, j} U_{n, j}(x), l_{n, i}^{\prime}(t) \psi(x)\right) d t \\
\quad+\int_{I_{n}}\left(c D_{0, x}^{\beta} a\left(\sum_{k=1}^{q} l_{n, k} U_{n, k}(x)\right) \sum_{j=1}^{q} l_{n, j} U_{n, j}(x), c D_{x, b}^{\beta}\left(l_{n, i}(t) \psi(x)\right)\right) d t \\
\quad+\int_{I_{n}}\left(c D_{0, x}^{\beta} b\left(\sum_{k=1}^{q} l_{n, k} U_{n, k}(x)\right) \sum_{j=1}^{q} l_{n, j} U_{n, j}(x), l_{n, i}(t) \psi(x)\right) d t \\
=\left(l_{n, 1}\left(t_{n, 1}\right) U_{n, 1}, l_{n, i}\left(t_{n, 1}\right) \psi(x)\right) .
\end{aligned}
$$

On time slab $I_{n}=\left(t_{n}, t_{n+1}\right]$, we define a Lagrange interpolation operator $\widehat{I}_{n}: C\left(I_{n}\right) \rightarrow$ $P_{q-1}\left(I_{n}\right)$, such that

$$
\widehat{I}_{n} y\left(t_{n, j}\right)=y\left(t_{n, j}\right), \quad j=1,2, \ldots, q,
$$

where the interpolation points are Radau points. It is easy to see that for given $x \in \Omega$, $\widehat{I}_{n} u(x, t) \in P_{q-1}\left(T_{n}\right)$ and $\widehat{I}_{n} u(x, t)=u\left(x, t_{n+1}\right)$ are available. On time slab $I_{n}=\left(t_{n}, t_{n+1}\right]$, $t_{n, 1}=t_{n}, t_{n, q}=t_{n+1}$, with the definition of interpolation operator, the discontinuous Galerkin scheme can be rewritten in detail as follows:

$$
\begin{aligned}
\delta_{q i}\left(U_{n, q}, \psi(x)\right)-\int_{I_{n}}\left(\sum_{j=1}^{q} l_{n, j} U_{n, j}(x), l_{n, i}^{\prime}(t) \psi(x)\right) d t \\
\quad+\int_{I_{n}}\left(c D_{0, x}^{\beta} a\left(\sum_{k=1}^{q} l_{n, k} U_{n, k}(x)\right) \sum_{j=1}^{q} l_{n, j} U_{n, j}(x){ }_{, C} D_{x, b}^{\beta}\left(l_{n, i}(t) \psi(x)\right)\right) d t \\
\quad+\int_{I_{n}}\left(c D_{0, x}^{\beta} b\left(\sum_{k=1}^{q} l_{n, k} U_{n, k}(x)\right) \sum_{j=1}^{q} l_{n, j} U_{n, j}(x), l_{n, i}(t) \psi(x)\right) d t \\
=l_{n, i}\left(t_{n}\right)\left(U_{n}, \psi(x)\right) .
\end{aligned}
$$

Next we introduce a lemma which is useful to prove the existence and estimate the error. 
Consider a $q \times q$ matrix $W$,

$$
W_{i j}=w_{n, j} l_{n, i}^{\prime}\left(t_{n, j}\right)=w_{j} l_{i}^{\prime}\left(\tau_{j}\right), \quad M=e_{q} e_{q}^{T}-N,
$$

where $e_{q}=(0,0, \ldots, 1)$.

It is clear that $W, M$ are independent of $K_{n}$. And if $Y^{T}=\left(y_{n, 1}, \ldots, y_{n, q}\right) \in R^{q}$, then

$$
Y^{T} M Y=\sum_{j=1}^{q} \delta_{q i} y_{n, q} y_{n, i}-\sum_{j=1}^{q} w_{n, j} l_{n, i}^{\prime}\left(t_{n, j}\right) y_{n, j} y_{n, i} .
$$

Lemma 3.2 (see [27]). Let $\widetilde{M}, D$ be the matrixes

$$
\widetilde{M}=D^{-1 / 2} M D^{1 / 2}, \quad D=\operatorname{diag}\left\{\tau_{1}, \ldots, \tau_{q}\right\} .
$$

If $\lambda=\left(\alpha_{0} / 2\right) \min \left\{w_{1} / \tau_{1}, w_{2} / \tau_{2}, \ldots, w_{q-1} / \tau_{q-1}, 1+w_{q}\right\}>0$, then there holds

$$
X^{T} \widetilde{M} X \geq \lambda|X|^{2}, \quad \forall X \in R^{q},
$$

where $|x|=\sum_{j=1}^{q} X_{i}^{2}$. $U$ by

In order to prove the existence and uniqueness, we need to define a new exhibition for

$$
U=\sum_{j=1}^{q} \tau_{j}^{1 / 2} l_{n, j}(T) U_{n, j}(x), \quad(x, t) \in \Omega \times I_{n}
$$

where $\tilde{U}=\tau_{j}^{-1 / 2} U_{n, j} \in S_{h}^{n}$. Then $\left.U\right|_{I_{n}}$ is uniquely determined by the function $\tilde{U}=\tilde{U}(x) \in S_{h}^{n}$. We choose $\psi=\tau_{i}^{-1 / 2} \phi$, where $\phi \in S_{h^{\prime}}^{n}$ and use the new expression $U$ to obtain the following results:

$$
\begin{aligned}
\delta_{q i}(\tilde{U}, \phi) & +\int_{I_{n}}\left(-\sum_{j=1}^{q} \tau_{j}^{1 / 2} l_{n, j} \tilde{U}_{n, j}, \sum_{k=1}^{q} \tau_{i}^{-1 / 2} l_{n, k} l_{n, i}^{\prime}\left(t_{n, k}\right) \phi\right) d t \\
& +\int_{I_{n}}\left(\sum_{j=1}^{q} \tau_{j}^{1 / 2} c D_{0, x}^{\beta} a\left(\sum_{m=1}^{q} \tau_{m}^{1 / 2} l_{n, m} \tilde{U}_{n, m}\right) l_{n, j} \tilde{U}_{n, j}, c D_{x, b}^{\beta} \tau_{i}^{-1 / 2} l_{n, i} \phi\right) d t \\
& +\int_{I_{n}}\left(\sum_{j=1}^{q} \tau_{j}^{1 / 2} c D_{0, x}^{\beta} b\left(\sum_{m=1}^{q} \tau_{m}^{1 / 2} l_{n, m} \tilde{U}_{n, m}\right) l_{n, j} \tilde{U}_{n, j}, \tau_{i}^{-1 / 2} l_{n, i} \phi\right) d t \\
= & l_{n, i}\left(t_{n}^{+}\right) \tau^{-1 / 2}\left(\tilde{U}_{n}, \phi\right) .
\end{aligned}
$$


Set that $a(\tilde{U})=a\left(\sum_{m=1}^{q} \tau_{m}^{1 / 2} l_{n, m} \tilde{U}_{n, m}\right)$ and $W_{n, j}=\int_{I_{n}} l_{n, j}^{2}(t) d t$, the above equation can be rewritten as

$$
\begin{aligned}
& -\sum_{j=1}^{q} W_{n, j} \tau_{j}^{1 / 2} \tau_{i}^{-1 / 2} l_{n, i}^{\prime}\left(t_{n, j}\right)\left(\tilde{U}_{n, j}, \phi\right)+\sum_{j=1}^{q} W_{n, j} \tau_{j}^{1 / 2} \tau_{i}^{-1 / 2}\left({ }_{c} D_{0, x}^{\beta} a(\tilde{U}) \tilde{U}_{n, j}, c D_{x, b}^{\beta} \phi\right) \\
& \quad+\sum_{j=1}^{q} W_{n, j} \tau_{j}^{1 / 2} \tau_{i}^{-1 / 2}\left(c D_{0, x}^{\beta} b(\tilde{U}) \tilde{U}_{n, j}, \phi\right)+\delta_{q i}(\tilde{U}, \phi) \\
& =l_{n, i}\left(t_{n}^{+}\right) \tau^{-1 / 2}\left(\tilde{U}_{n}, \phi\right) .
\end{aligned}
$$

Theorem 3.3. Let $U_{n}$ be given in $S_{h^{\prime}}^{n}$ then for the sufficiently small $k_{n}$, there exists $\left\{\tilde{U}_{n, j}\right\}_{j=1}^{q} \in S_{h}^{n}$ satisfying (3.32); hence, (3.17) has a unique solution $U \in T_{h k}$.

Proof of Theorem 3.3. The vector space $\left(S_{h}^{n}\right)^{q}$ is a Hilbert space with finite dimension. For all $x=\left(x_{1}, x_{2}, \ldots, x_{q}\right)^{T}, \phi=\left(\phi_{1}, \phi_{2}, \ldots, \phi_{q}\right)^{T} \in S_{h}^{n}$ equipped with the norm

$$
\||x|\|=\left(\sum_{j=1}^{q}\left\|x_{i}^{2}\right\|\right)^{1 / 2}, \quad\||x|\|_{\beta}=\left(\sum_{j=1}^{q}\left\|x_{i}^{2}\right\|_{\beta}\right)^{1 / 2}
$$

Define a map $F$ from $\left(S_{h}^{n}\right)^{q}$ to itself by

$$
\begin{aligned}
\left(F(v)_{i}, \phi\right)= & \left(v_{i}, \phi\right)+\delta_{q i}\left(v_{q}, \phi\right)-\sum_{j=1}^{q} W_{n, j} \tau_{j}^{1 / 2} \tau_{i}^{-1 / 2} l_{n, i}^{\prime}\left(v_{i}, \phi\right) \\
& +W_{n, i}\left(c D_{0, x}^{\beta}\left(a(v) v_{i}\right), c D_{x, b}^{\beta} \phi\right)+W_{n, i}\left(c D_{0, x}^{\beta} b(v) v_{i}, \phi\right)-\left(U_{n}, \tau_{i}^{-1 / 2} l_{n, i}\left(t_{n}\right) \phi\right) .
\end{aligned}
$$

Since

$$
\left(v_{i}, \phi\right)+\delta_{q i}\left(v_{q}, \phi\right)-\sum_{j=1}^{q} W_{n, j} \tau_{j}^{1 / 2} \tau_{i}^{-1 / 2} l_{n, i}^{\prime}\left(v_{i}, \phi\right)-\left(U_{n}, \tau_{i}^{-1 / 2} l_{n, i}\left(t_{n}\right) \phi\right)
$$

is a linear system, $a(x, t, u), b(x, t, u)$ are all continuous maps, and the map $F$ is a continuous map from $\left(S_{h}^{n}\right)^{q}$ into itself. So according to the Brouwer fixed point theorem, there exists at least one fixed point $v_{i}$ such that $\left(F(v)_{i}, \phi\right)=\left(v_{i}, \phi\right)$. So $(3.32)$ has at least one solution, denoted by $v$. Next we investigate the uniqueness of the solution. 
Let $v$ and $v^{*}$ be two solutions of (3.32), setting $\phi=v_{i}-v_{i}^{*}$ and summing $i$ from 1 to $q$, then we have

$$
\begin{aligned}
\sum_{i=1}^{q}\left(F(v-v *)_{i}, v_{i}-v_{i}^{*}\right) \\
=\delta_{q i}\left(v_{q}-v_{q}^{*}, v_{i}-v_{i}^{*}\right)-\sum_{i=1}^{q} \sum_{j=1}^{q} W_{n, j} l_{n, i}^{\prime}\left(t_{n, j}\right) \tau_{j}^{1 / 2} \tau_{i}^{-1 / 2}\left(v_{j}-v_{j}^{*}, v_{i}-v_{i}^{*}\right) \\
\quad+\sum_{i=1}^{q} \sum_{j=1}^{q} W_{n, j} \tau_{j}^{1 / 2} \tau_{i}^{-1 / 2}\left(c D_{0, x}^{\beta} a\left(v-v^{*}\right)\left(v_{j}-v_{j}^{*}\right), c D_{x, b}^{\beta}\left(v_{i}-v_{i}^{*}\right]\right) \\
+\sum_{i=1}^{q} \sum_{j=1}^{q} W_{n, j} \tau_{j}^{1 / 2} \tau_{i}^{-1 / 2}\left(c D_{0, x}^{\beta} b\left(v-v^{*}\right)\left(v_{j}-v_{j}^{*}\right),\left(v_{i}-v_{i}^{*}\right)\right) \\
\quad+\sum_{i=1}^{q}\left(v_{i}-v_{i}^{*}, v_{i}-v_{i}^{*}\right) .
\end{aligned}
$$

According to Lemma 3.2, we can see that for the first two terms of the right-hand side of (3.36), there exists a constant $\alpha$, such that

$$
\begin{gathered}
\delta_{q i}\left(v_{q}-v_{q}^{*}, v_{i}-v_{i}^{*}\right)-\sum_{i=1}^{q} \sum_{i=1}^{q} W_{n, j} l_{n, i}^{\prime}\left(t_{n, j}\right) \tau_{j}^{1 / 2} \tau_{i}^{-1 / 2}\left(v_{j}-v_{j}^{*}, v_{i}-v_{i}^{*}\right) \\
=\left(\widetilde{M}\left(v-v^{*}\right), v-v^{*}\right) \geq \alpha\left\|v-v^{*}\left|\left\|^{2} \geq C \alpha\right\|\right| v-v^{*} \mid\right\|_{\beta}^{2} .
\end{gathered}
$$

With the help of bounded assumption of $a$ and $b$, we have

$$
\begin{aligned}
& \sum_{i=1}^{q} \sum_{j=1}^{q} W_{n, j} \tau_{j}^{1 / 2} \tau_{i}^{-1 / 2}\left({ }_{c} D_{0, x}^{\beta} a\left(v-v^{*}\right)\left(v_{j}-v_{j}^{*}\right),{ }_{C} D_{x, b}^{\beta}\left[\left(v_{i}-v_{i}^{*}\right)\right]\right) \\
& \quad \leq \sum_{i=1}^{q} \int_{I_{n}}\left|\left({ } D_{0, x}^{\beta} a\left(v-v^{*}\right) \sum_{j=1}^{q} \tau_{j}^{1 / 2} \tau_{i}^{-1 / 2} l_{n, j}(t)\left(v_{j}-v_{j}^{*}\right), l_{n, i}(t){ }_{C} D_{x, b}^{\beta}\left(v_{i}-v_{i}^{*}\right)\right)\right| d t \\
& \quad \leq \sum_{i, j=1}^{q} M \int_{I_{n}}\left|\tau_{j}^{1 / 2} \tau_{i}^{-1 / 2} l_{n, i}(t) l_{n, j}(t)\left(c D_{0, x}^{\beta}\left(v_{j}-v_{j}^{*}\right),{ }_{C} D_{x, b}^{\beta}\left(v_{i}-v_{i}^{*}\right)\right)\right| d t \\
& \quad \leq C k_{n}\left|\left\|v_{k}-v_{k}^{*}\right\|\right|_{\beta^{\prime}}^{2} \\
& \sum_{i=1}^{q} \sum_{j=1}^{q} W_{n, j} \tau_{j}^{1 / 2} \tau_{i}^{-1 / 2}\left(c D_{0, x}^{\beta} b\left(v-v^{*}\right)\left(v_{j}-v_{j}^{*}\right),\left(v_{i}-v_{i}^{*}\right)\right) \\
& \quad \leq \sum_{i=1}^{q} \int_{I_{n}}\left|\left(\sum_{j=1}^{q} \tau_{j}^{1 / 2} \tau_{i}^{-1 / 2} l_{n, j}(t){ }_{C} D_{0, x}^{\beta} b\left(v-v^{*}\right)\left(v_{j}-v_{j}^{*}\right), l_{n, i}(t)\left(v_{i}-v_{i}^{*}\right)\right)\right| d t \\
& \quad \leq \sum_{i, j=1}^{q} M \int_{I_{n}}\left|\tau_{j}^{1 / 2} \tau_{i}^{-1 / 2} l_{n, i}(t) l_{n, j}(t){ }_{C} D_{0, x}^{\beta}\left(v_{j}-v_{j}^{*}\right),\left(v_{i}-v_{i}^{*}\right)\right| d t .
\end{aligned}
$$


According to the boundedness of fractional operator ${ }_{C} D_{0, x}^{\beta}$, inequality $\left\|_{C} D_{0, x}^{\beta}\left(v_{i}-v_{i}^{*}\right)\right\| \leq$ $C\left\|v_{i}-v_{i}^{*}\right\|$ holds. Furthermore, we have

$$
\begin{aligned}
& \sum_{i, j=1}^{q} M \int_{I_{n}}\left|\tau_{j}^{1 / 2} \tau_{i}^{-1 / 2} l_{n, i}(t) l_{n, j}(t)\left(c D_{0, x}^{\beta}\left(v_{j}-v_{j}^{*}\right),\left(v_{i}-v_{i}^{*}\right)\right)\right| d t \\
& \quad \leq \sum_{j=1}^{q} \tau_{j}^{1 / 2} \tau_{j}^{-1 / 2}\left\|W_{n, k}\left(v_{k}-v_{k}^{*}, v_{k}-v_{k}^{*}\right)\right\| \\
& \quad=M \sum_{k=1}^{q} W_{n, k}\left\|v_{k}-v_{k}^{*}\right\|^{2} \\
& \quad \leq C k_{n}\left|\left\|v_{k}-v_{k}^{*}\right\|\right|_{\beta}^{2} .
\end{aligned}
$$

The fifth term of the right-hand side of (3.36) is estimated in

$$
\sum_{j=1}^{q}\left(v_{i}-v_{i}^{*}, v_{i}-v_{i}^{*}\right)=\left.\left|\| v-v^{*}\right|\right|^{2} \leq\left.||\left|v-v^{*}\right|\right|_{\beta} ^{2} .
$$

And by the Brouwer fixed point theorem, one gets

$$
\left(F\left(v-v^{*}\right)_{i}, v_{i}-v_{i}^{*}\right)=\left(v_{i}-v_{i}^{*}, v_{i}-v_{i}^{*}\right)
$$

furthermore,

$$
\sum_{i=1}^{q}\left(F\left(v-v^{*}\right)_{i}, v_{i}-v_{i}^{*}\right)=\sum_{i=1}^{q}\left(v_{i}-v_{i}^{*}, v_{i}-v_{i}^{*}\right)=\left.\left|\| v_{i}-v_{i}^{*}\right|\right|^{2}
$$

By using inequalities (3.37)-(3.40) and (3.42), one has

$$
\left.C\left(\alpha-k_{n}\right)||\left|v_{i}-v_{i}^{*}\right|\right|_{\beta} ^{2} \leq 0 .
$$

Choosing $\alpha \geq k_{n}$, we have

$$
\|\left.\left|v_{i}-v_{i}^{*}\right|\right|_{\beta} ^{2} \leq 0
$$

That is impossible. So the uniqueness is proved.

\section{Error Estimation}

Now we turn to analyze the error estimate of the D-G scheme. 
Let $u_{h}^{n}$ be the approximate solution on time slab $I_{n}$. Denote $P_{h} u^{n}$ as a Ritz-Galerkin projection operator defined as follows:

$$
\begin{aligned}
& \left({ }_{c} D_{0, x}^{\beta} a(u)\left(u^{n}-P_{h} u^{n}\right),{ }_{c} D_{x, b}^{\beta} v\right)=0, \\
& \left({ }_{c} D_{0, x}^{\beta} a\left(u_{0}\right)\left(u^{n}-P_{h} u^{n}\right),{ }_{c} D_{x, b}^{\beta} v\right)=0 .
\end{aligned}
$$

And $\rho^{n}=P_{h} u^{n}-u^{n}, \theta^{n}=u_{h}^{n}-W^{n}$, then one has

$$
u_{h}^{n}-u^{n}=u_{h}^{n}-W^{n}+W^{n}-u^{n}=\theta^{n}+W^{n}-u^{n},
$$

where $W: I \rightarrow H_{0}^{\beta}(\Omega)$ is defined by

$$
W(x, t)=\widehat{I}_{n}^{q} P_{h} u(x, t), \quad(x, t) \in \Omega \times I_{n} .
$$

It can be seen that $W$ is an element of $T_{h k}^{n}$.

Lemma 4.1. Let $a(u), b(u)$ be smooth functions on $\Omega$, and $0<m \leq a(u), b(u) \leq M, 0<\beta<1$, and $P_{h} u^{n}$ is defined as above, then

$$
\left\|P_{h} u^{n}-u^{n}\right\| \leq c_{2} h_{n}^{1+\beta}\|u\|_{1+\beta} .
$$

Proof of Lemma 4.1. For all $\phi \in L^{2}(\Omega)$, $w$ is the solution of the following equation:

$$
\begin{gathered}
-a(w)_{C} D_{0, x}^{2 \beta} w=\phi, \quad w \in \Omega, \\
w=0, \quad w \in \partial \Omega .
\end{gathered}
$$

So the next equation holds

$$
\|w\|_{2 \beta} \leq c_{2}\|\phi\| .
$$

And for all $x \in S_{h}$, by the aid of approximation properties of $S_{h}$ and the weak form, we can derive

$$
\begin{aligned}
\left(P_{h} u^{n}-u^{n}, \phi\right) & =-\left(P_{h} u^{n}-u^{n}, a(w)_{c} D_{0, x}^{2 \beta} w\right)=\left(c D_{x, b}^{\beta} a(w)\left(P_{h} u^{n}-u^{n}\right), c D_{0, x}^{\beta} w\right) \\
& =\left({ }_{c} D_{x, b}^{\beta} a(w)\left(P_{h} u^{n}-u^{n}\right), c D_{0, x}^{\beta}(w-x)\right) \leq M\left\|P_{h} u^{n}-u^{n}\right\|_{\beta}\|w-x\|_{\beta} \\
& \leq M\left\|P_{h} u^{n}-u^{n}\right\|_{\beta} \inf _{x \in S_{h}}\|w-x\|_{\beta} \\
& \leq c_{2} h^{r-\beta}\|u\|_{r} h^{\beta}\|w\|_{\beta}=c_{2} h^{r}\|u\|_{r}\|\phi\| .
\end{aligned}
$$


So

$$
\left\|P_{h} u^{n}-u^{n}\right\|=\sup _{0 \neq \phi \in L^{2}(\Omega)} \frac{\left(P_{h} u^{n}-u^{n}, \phi\right)}{\|\phi\|} \leq c_{2} h^{r}\|u\|_{r}
$$

Lemma 4.2. Let $P_{h} u^{n}$ be defined as (4.1), then $\left\|D\left(P_{h} u^{n}\right)\right\|$ and $\left\|_{C} D_{0, x}^{\beta} P_{h} u^{n}\right\|$ are bounded, where $0 \leq \beta \leq 1$.

Proof of Lemma 4.2. Using the properties of the norm, then the following inequality is valid:

$$
\left\|{ }_{C} D_{0, x}^{\beta} P_{h} u^{n}\right\|_{L^{\infty}} \leq\left\|{ }_{C} D_{0, x}^{\beta}\left(P_{h} u^{n}-I_{h} u^{n}\right)\right\|_{L^{\infty}}+\left\|c D_{0, x}^{\beta}\left(I_{h} u^{n}\right)\right\|_{L^{\infty}} .
$$

According to the estimate of interpolation, we get

$$
\left\|c D_{0, x}^{\beta}\left(P_{h} u^{n}-I_{h} u^{n}\right)\right\|_{L^{\infty}} \leq c\left(\left\|c D_{0, x}^{\beta} \rho^{n}\right\|_{L^{\infty}}+\left\|c D_{0, x}^{\beta}\left(I_{h} u^{n}-u^{n}\right)\right\|_{L^{\infty}}\right) .
$$

From Lemmas 2.8 and 4.1, and the inverse estimate, we know that $\left\|{ }_{C} D_{0, x}^{\beta} \rho^{n}\right\|_{L^{\infty}} \leq c\left\|u^{n}\right\|$. Therefore, it is easy to see that

$$
\begin{gathered}
\left\|{ }_{C} D_{0, x}^{\beta}\left(I_{h} u^{n}\right)\right\|_{L^{\infty}} \leq c\left\|c D_{0, x}^{\beta} u^{n}\right\|_{L^{\infty}} \\
\left\|c D_{0, x}^{\beta}\left(I_{h} u^{n}-u^{n}\right)\right\|_{L^{\infty}} \leq c\left\|c D_{0, x}^{\beta} u^{n}\right\|_{L^{\infty}} .
\end{gathered}
$$

So

$$
\left\|c D_{0, x}^{\beta}\left(P_{h} u^{n}\right)\right\|_{L^{\infty}} \leq c\left\|c D_{0, x}^{\beta} u^{n}\right\|_{L^{\infty}}+c\left\|u^{n}\right\| .
$$

The boundedness $\left\|{ }_{C} D_{0, x}^{\beta} P_{h} u^{n}\right\|$ can be derived from above. The proof of $\left\|D P_{h} u^{n}\right\| \leq c(u)$ can be similarly given.

Let $\widehat{I}_{n}^{q}: C\left(I_{n}\right) \rightarrow P_{q}\left(I_{n}\right)$ be the usual Lagrange interpolation operator at the Radau points on $I_{n}=\left(t_{n}, t_{n+1}\right]$; that is,

$$
\left(\widehat{I}_{n}^{q} y\right)\left(t_{n, j}\right)=y\left(t_{n, j}\right), \quad j=1,2, \ldots, q
$$

where $t_{n, j}, j=1,2, \ldots, q$ are the Radau points. Therefore, we can see that

$$
\begin{gathered}
\left(\widehat{I}_{n}^{q} u\right)(x, \cdot) \in P_{q-1}\left(I_{n}\right), \\
\left(\widehat{I}_{n}^{q} u\right)\left(x, t_{n+1}\right)=u\left(x, t_{n+1}\right), \quad x \in \Omega .
\end{gathered}
$$


Lemma 4.3. The interpolation operation $\widehat{I}_{n}^{q}$ on $P_{h}\left(I_{n}\right)$ has the following property:

$$
\left\|\widehat{I}_{n}^{q} u-u\right\| \leq c h^{s}\|u\|_{s}, \quad \forall u \in H^{s} \cap H_{0}^{\beta} .
$$

Lemma 4.4. For $W$ defined above, we have the following error estimates:

$$
\begin{gathered}
\max _{I_{n}}\|u-W\|_{\beta} \leq C k_{n}^{q} \max _{I_{n}}\left\|u^{(q)}\right\|_{\beta}+c h^{s-\beta} \max _{I_{n}}\|u\|_{s}, \quad 2 \leq s \leq r+1, \\
\|u-W\|_{0, \beta, n} \leq C k_{n}^{q}\left\|u^{(q)}\right\|_{0,0, n}+C h_{n}^{s-\beta}\|u\|_{0, s, n}, \quad 2 \leq s \leq r .
\end{gathered}
$$

Proof of Lemma 4.4. Since

$$
\|u-W\|_{\beta}=\left\|u-P_{h} u+P_{h} u-W\right\|_{\beta} \leq\left\|u-P_{h} u\right\|_{\beta}+\left\|P_{h} u-W\right\|_{\beta}
$$

according to Lemma 4.1, we have

$$
\begin{aligned}
\left\|u-P_{h} u\right\|_{\beta}+\left\|P_{h} u-W\right\|_{\beta} & \leq h^{s-\beta}\|u\|_{s}+\left\|P_{h} u-W\right\|_{\beta} \\
& \leq h^{s-\beta}\|u\|_{s}+C k_{n}^{q}\left\|\left(P_{h} u\right)^{(q)}\right\|_{\beta} \leq h^{s-\beta}\|u\|_{s}+C k_{n}^{q}\left\|u^{(q)}\right\|_{\beta} \\
& \leq h^{s-\beta}\|u\|_{s}+C k_{n}^{q}\left\|u^{(q)}\right\|_{\beta} .
\end{aligned}
$$

The first inequality can be similarly proved.

We are now ready to prove convergence result. Putting $W$ into D-G finite element formula (3.17), and setting $\theta=U-W$, we can get the basic error equation as follows:

$$
\begin{aligned}
& \left(\theta_{n+1}^{-}, v_{n+1}^{-}\right)-\left(\theta_{n}^{-}, v_{n}^{+}\right)-\int_{I_{n}}\left(\theta, v_{t}\right) d t+\int_{I_{n}}\left(c D_{0, x}^{\beta} a(U) \theta-b(U)(U-W), c D_{x, b}^{\beta} v\right) d t \\
& =-\left(W_{n+1}^{-}, v_{n+1}^{-}\right)+\left(W_{n}^{-}, v_{n}^{+}\right)+\int_{I_{n}}\left(W, v_{t}\right) d t-\int_{I_{n}}\left({ }_{c} D_{0, x}^{\beta} a(U) W+b(U) W, c D_{x, b}^{\beta} v\right) d t
\end{aligned}
$$

where $W_{0}=P_{h} u_{0}$ and $\theta_{0}=U_{0}-W_{0}=u_{0}-P_{h} u^{0}$.

Theorem 4.5. Let $u$ be the solution of Problem 1. If $U$ is a solution of the discontinuous Galerkin scheme (3.17), then the following error estimation holds:

$$
\begin{aligned}
\|u-U\|_{\infty, \beta}= & C \max _{0 \leq n \leq N-1} h^{s} \max _{I_{n}}\left(\left\|u_{t}\right\|_{s}+\|u\|_{s}+h^{-\beta}\|u\|_{s}\right) \\
& +C \max _{0 \leq n \leq N-1}\left\{k_{n}^{q} \max _{I_{n}}\left(\left\|u^{(q+1)}\right\|+\left\|u^{(q)}\right\|+\left\|D_{x}^{2 \beta} u^{(q)}\right\|+\left\|D_{x}^{\beta} u^{(q)}\right\|\right)\right\},
\end{aligned}
$$

where $2 \leq s \leq r$. 
Proof of Theorem 4.5. It is necessary to rewrite $W$ and discrete error $\theta$ as follows:

$$
\begin{gathered}
W=\sum_{j=1}^{q} l_{n, j}(t) P_{h} u_{n, j}, \\
\theta=U-W=\sum_{j=1}^{q} l_{n, j}(t)\left(U_{n, j}-P_{h} u_{n, j}\right),
\end{gathered}
$$

where $u_{n, j}=u\left(x, t_{n, j}\right)$. We also denote $a\left(\sum_{j=1}^{q} l_{n, j}(t) U_{n, j}\right), b\left(\sum_{j=1}^{q} l_{n, j}(t) U_{n, j}\right)$ as $a(U)$ and $b(U)$. Noting that $t_{n, q}=t_{n+1}, t_{n, 1}=t_{n}$, so $l_{n, q}\left(t_{n, q}\right)=1$ and $l_{n, 1}\left(t_{n, 1}\right)=1$. Setting $W_{n, i}=\int_{I_{n}} l_{n, i}^{2}(t) d t$ and $v=l_{n, i}(t) \phi(x), \phi \in S_{h}^{n}$, then the basic error equation can be rearranged as follows:

$$
\begin{aligned}
-\delta_{q i}( & \left.P_{h} u_{n, q}, \phi\right)+l_{n, i}\left(t_{n}\right)\left(P_{h} u_{n}, \phi\right)+\left(\sum_{j=1}^{q} W_{n, j} l_{n, i}^{\prime}\left(t_{n, j}\right) P_{h} u_{n, j}, \phi\right)-\left(u_{n}^{-}, l_{n, i}\left(t_{n}\right) \phi\right) \\
& -\left(\sum_{j=1}^{q} W_{n, j} c D_{0, x}^{\beta} a(U) P_{h} u_{n, j}, c D_{x, b}^{\beta} \phi\right)+\sum_{j=1}^{q} W_{n, j}\left(b(U)(t) P_{h} u_{n, j}, c D_{x, b}^{\beta} \phi\right) \\
& -\int_{I-n}\left(u, l_{n, i}^{\prime}(t) \phi\right) d t+\int_{I_{n}}\left(c D_{0, x}^{\beta}(a(u) u)+b(u) u, l_{n, i}(t) c D_{x, b}^{\beta} \phi\right) d t \\
& +\left(u_{n+1}^{-}, l_{n, i}\left(t_{n+1}\right) \phi\right) \\
= & \left(R_{1}, \phi\right)+\left(R_{2}, \phi\right)+\left(R_{3, c} D_{x, b}^{\beta} \phi\right)+\left(R_{4}, c D_{x, b}^{\beta} \phi\right)+\left(R_{5}, \phi\right),
\end{aligned}
$$

where

$$
\begin{aligned}
& R_{1}=\sum_{j=1}^{q} W_{n, j} l_{n, i}^{\prime}\left(t_{n, j}\right)\left(P_{h} u_{n, j}-u_{n, j}\right)-\delta_{q i}\left(P_{h} u_{n, q}-u_{n, q}\right)-l_{n, i}\left(t_{n}\right)\left(u_{n}^{+}-P_{h} u_{n}^{+}\right), \\
& R_{2}=\sum_{j=1}^{q} W_{n, j} l_{n, i}^{\prime}\left(t_{n, j}\right) u_{n, j}-\int_{I-n} l_{n, i}^{\prime}(t) u d t, \\
& R_{3}=-\sum_{j=1}^{q} W_{n, j} C D_{0, x}^{\beta} a(U) P_{h} u_{n, j}+\int_{I_{n}} l_{n, i}(t) c D_{x, b}^{\beta} a(u) u d t, \\
& R_{4}=-\sum_{j=1}^{q} W_{n, j} b(U)\left(P_{h} u_{n, j}\right)+\int_{I_{n}} l_{n, i}(t) b(u) u d t, \\
& R_{5}=l_{n, i}\left[u_{n}-P_{h} u_{n}\right]=l_{n, i}\left(t_{n}\right)\left\{\left(u_{n}^{+}-P_{h} u_{n}^{+}\right)-\left(u_{n}^{-}-P_{h} u_{n}^{-}\right)\right\} .
\end{aligned}
$$


Let $\phi=\theta_{n, i}$ and sum $i$ from 1 to $q$, then the error equation can be derived as

$$
\begin{gathered}
\left(\theta_{n+1}^{-}, \theta_{n+1}^{-}\right)-\left(\theta_{n}^{-}, \theta_{n}^{+}\right)-\int_{I_{n}}\left(\theta, \theta_{t}\right) d t+\int_{I_{n}}\left(c D_{0, x}^{\beta} a(U) \theta, c D_{x, b}^{\beta} \theta\right) d t+\int_{I_{n}}\left(b(U) \theta, c D_{x, b}^{\beta} \theta\right) d t \\
=\sum_{i=1}^{q}\left(R_{1}, \theta_{n, i}\right)+\left(R_{2}, \theta_{n, i}\right)+\left(R_{3}, c D_{x, b}^{\beta} \theta_{n, i}\right)+\left(R_{4}, c D_{x, b}^{\beta} \theta_{n, i}\right)+\left(R_{5}, \theta_{n, i}\right) .
\end{gathered}
$$

Note that

$$
-\int_{I_{n}}\left(\theta, \theta_{t}\right) d t=-\frac{1}{2} \int_{I_{n}} \frac{d}{d t}\|\theta\|^{2}=-\frac{1}{2}\left\|\theta_{n+1}^{-}\right\|^{2}+\frac{1}{2}\left\|\theta_{n}^{+}\right\|^{2}
$$

Consider the bounded assumption of $a(u)$ and $b(u)$; the following inequality can be got:

$$
\int_{I_{n}}\left({ }_{c} D_{0, x}^{\beta} a(U) \theta, c D_{x, b}^{\beta} \theta\right) d t \geq m \int_{I_{n}}\left(c D_{0, x}^{\beta} \theta, c D_{x, b}^{\beta} \theta\right) d t=m \int_{I_{n}}\left\|{ }_{c} D_{0, x}^{\beta} \theta\right\|^{2} d t .
$$

Since $b(u)$ is bounded, and from Lemma 2.8, where ${ }_{C} D_{x, b}^{\beta}$ is a linear bounded map, the following inequality is established:

$$
\begin{aligned}
\int_{I_{n}}\left(b(U) \theta, c D_{x, b}^{\beta} \theta\right) d t & \geq m \int_{I_{n}}\left(\theta, c D_{x, b}^{\beta} \theta\right) d t \\
& \geq C m \int_{I_{n}}\|\theta\|\left\|{ }_{C} D_{x, b}^{\beta} \theta\right\| d t \geq C m \int_{I_{n}}\|\theta\|^{2} d t .
\end{aligned}
$$

The first and second terms of the left side of (4.24) can be seen as

$$
\begin{gathered}
\left(\theta_{n+1}^{-}, \theta_{n+1}^{-}\right)=\left\|\theta_{n+1}^{-}\right\|^{2}, \\
\left(\theta_{n}^{-}, \theta_{n}^{+}\right) \leq\left\|\theta_{n}^{-}\right\| \cdot\left\|\theta_{n}^{+}\right\| \leq\left\|\theta_{n}^{-}\right\|^{2}+\frac{\left\|\theta_{n}^{+}\right\|^{2}}{4} .
\end{gathered}
$$

Taking (4.24)-(4.27) into the left side of (4.22) yields

$$
\begin{aligned}
\frac{1}{2} \| \theta_{n+1}^{-} & \left\|^{2}+\frac{1}{2}\right\| \theta_{n}^{+}\left\|^{2}+m \int_{I_{n}}\right\| c D_{0, x}^{\beta} \theta\left\|^{2} d t+C m \int_{I_{n}}\right\| \theta \|^{2} d t \\
\leq & \left\|\theta_{n}^{-}\right\|^{2}+\frac{\left\|\theta_{n}^{+}\right\|^{2}}{4} \\
& +\sum_{i=1}^{q}\left\{\left(R_{1}, \theta_{n, i}\right)+\left(R_{2}, \theta_{n, i}\right)+\left(R_{3, c} D_{x, b}^{\beta} \theta_{n, i}\right)+\left(R_{4, c} D_{x, b}^{\beta} \theta_{n, i}\right)+\left(R_{5}, \theta_{n, i}\right)\right\} .
\end{aligned}
$$


As to $R_{1}$, for $i=1,2, \ldots, q$, there exists

$$
\delta_{q i}-\sum_{j=1}^{q} W_{n, j} l_{n, i}^{\prime}\left(t_{n, i}\right)-l_{n, i}\left(t_{n}^{+}\right)=\delta_{q i}-\int_{I_{n}} l_{n, i}^{\prime}(t) d t-l_{n, i}\left(t_{n}^{+}\right)=0 .
$$

This means that there exists constants $C_{i j}$ and $C$, which are independent of $n$, such that

$$
\begin{aligned}
\left\|R_{1}\right\| & =\left\|\sum_{j=1}^{q} C_{i j}\left(\rho_{n, j}-\rho_{n, j-1}\right)\right\| \leq\left\|\int_{I_{n}} C \rho_{t}(\tau) d \tau\right\| \\
& \leq C \int_{I_{n}} h^{s}\left\|u_{t}(\tau)\right\|_{s} d \tau \leq C h^{s} k_{n}^{1 / 2}\left\|u_{t}\right\|_{0, s, n} .
\end{aligned}
$$

In order to estimate $R_{2}$, we apply the interpolation operator. Let $\widehat{I}_{q}^{n}: C\left(\widehat{I}_{n}\right) \rightarrow P_{q}\left(\widehat{I}_{n}\right)$ be the interpolation operator on the time slab $I_{n}$, whose order is less than $q$. The interpolation points involve not only Radau points, but also $t_{n}$, and these points satisfy that $\widehat{I}_{n}^{q} y\left(t_{n, j}\right)=$ $y\left(t_{n, j}\right), \widehat{I}_{n}^{q} y\left(t_{n}^{+}\right)=y\left(t_{n}^{+}\right), j=1,2, \ldots, q$. Then, for every $x \in \Omega$, such that $l_{n, i}^{\prime} \widehat{I}_{n}^{q} u$ is a polynomial whose order is $2 q-2$,

$$
\begin{aligned}
\left\|R_{2}\right\| & =\left\|\sum_{j=1}^{q} W_{n, j} l_{n, i}^{\prime}\left(t_{n, j}\right) u_{n, j}-\int_{I_{n}} l_{n, i}^{\prime}(t) u d t\right\| \\
& =\left\|\int_{I_{n}} l_{n, i}^{\prime}(t)\left(\widehat{I}_{n}^{q} u-u\right) d t\right\| \leq C k_{n}^{q+1 / 2}\left\|u^{(q+1)}\right\|_{0,0, n} .
\end{aligned}
$$

As to $R_{3}$, when $k_{n}$ is sufficiently small, with the aid of Hölder inequality, we obtain

$$
\begin{aligned}
\left(R_{3, c} D_{x, b}^{\beta} \theta_{n, i}\right)= & -\left(c D_{0, x}^{\beta} R_{3}, \theta_{n, i}\right) \\
\leq & \left\|\sum_{j=1}^{q} W_{n, j} c D_{0, x}^{2 \beta}(a(U)-a(u)) u_{n, j}\right\| \cdot\left\|\theta_{n, i}\right\| \\
& +\left\|\sum_{j=1}^{q} W_{n, j} c D_{0, x}^{2 \beta} a(u) u_{n, j}-\int_{I_{n}} l_{n, i}(t)_{c} D_{0, x}^{2 \beta} a(u) u d t\right\| \cdot\left\|\theta_{n, i}\right\| \\
= & \left(\left\|R_{31}\right\|+\left\|R_{32}\right\|\right)\left\|\theta_{n, i}\right\|,
\end{aligned}
$$


where

$$
\begin{aligned}
\left\|R_{31}\right\| & =\left\|\sum_{j=1}^{q} W_{n, j} C D_{0, x}^{2 \beta}(a(U)-a(u)) u_{n, j}\right\| \\
& \leq \int_{I_{n}} l_{n, i}^{2}(t)\left\|c D_{0, x}^{2 \beta} \theta\right\|^{2} d t+L C \int_{I_{n}} l_{n, i}^{2}(t)\left\|c D_{0, x}^{2 \beta}(W-u)\right\| d t \\
& \leq L C \int_{I_{n}} l_{n, i}^{2}(t)\left\|c D_{0, x}^{2 \beta} \theta\right\|^{2} d t+C k_{n}^{q+1 / 2}\left\|c D_{0, x}^{2 \beta} u^{(q)}\right\|_{0,0, n} \\
& \leq L C \int_{I_{n}} l_{n, i}^{2}(t)\|\theta\|^{2} d t+C k_{n}^{q+1 / 2}\left\|c D_{0, x}^{2 \beta} u^{(q)}\right\|_{0,0, n^{\prime}} \\
\left\|R_{32}\right\| & =\left\|\sum_{j=1}^{q} W_{n, j} C D_{0, x}^{2 \beta} a(u) u_{n, j}-\int_{I_{n}} l_{n, i}(t)_{C} D_{0, x}^{2 \beta} a(u) u d t\right\| \\
& \leq M\left\|\sum_{j=1}^{q} W_{n, j} C D_{0, x}^{2 \beta} \widehat{I}_{n}^{q} u_{n, j}-\int_{I_{n}} l_{n, i}(t)_{C} D_{0, x}^{2 \beta} u d t\right\| \\
& \leq M\left\|\int_{I_{n}} l_{n, l}^{2}(t)\left(\widehat{I}_{n}^{q}-I\right)_{C} D_{0, x}^{2 \beta} u d t\right\| \\
& \leq C M k_{n}^{q+1 / 2}\left\|{ }_{C} D_{0, x}^{2 \beta} u^{(q)}\right\|_{0,0, n} .
\end{aligned}
$$

Almost similar to the estimation of $R_{3}$, the analysis of $R_{4}$ can be got by the help of boundedness of $b(u), u$, and $P_{h} u_{n}$

$$
\begin{aligned}
\left(R_{4}, c\right. & \left.D_{x, b}^{\beta} \theta_{n, i}\right) \\
\leq & \left\|\sum_{j=1}^{q} W_{n, j} c D_{0, x}^{\beta}(b(U)-b(u)) P_{h} u_{n, j}\right\|\left\|\theta_{n, i}\right\| \\
& +\left|\sum_{j=1}^{q} W_{n, j} C D_{0, x}^{\beta} b(u) P_{h} u_{n, j}-\int_{I_{n}} l_{n, i}(t)_{C} D_{0, x}^{\beta} b(u) u d t\right|\left\|\theta_{n, i}\right\| \\
\leq & C\left\{\int_{I_{n}} l_{n, j}\left\|C D_{0, x}^{\beta} \theta\right\| d t+k_{n}^{q+1 / 2}\left\|{ }_{C} D_{0, x}^{\beta} u^{(q)}\right\|_{0,0, n}+(M+L) h^{s-\beta} k_{n}^{1 / 2}\|u\|_{0, s, n}\right\}\left\|\theta_{n, i}\right\| .
\end{aligned}
$$

The last term contains $R_{5}$ which is estimated as follows:

$$
\begin{aligned}
\left\|\left(R_{5}, \theta_{n}^{+}\right)\right\| & \leq C\left\|\left(u_{n}^{+}-P_{h} u_{n}^{+}\right)\right\|^{2}+C\left\|u_{n}^{-}-P_{h} u_{n}^{-}\right\|^{2}+\frac{1}{4}\left\|\theta_{n}^{+}\right\|^{2} \\
& \leq C h_{n}^{2 s}\|u\|_{s}^{2}+C h_{n-1}^{2 s}\|u\|_{s}^{2}+\frac{1}{4}\left\|\theta_{n}^{+}\right\|^{2},
\end{aligned}
$$

where $h_{n}$ is the space partition in the time slab $I_{n}$. 
Taking the estimations from $R_{1}$ to $R_{5}$ into (4.29), and setting $W_{n, j}=k_{n} W_{j}$, since $\|\theta\|_{0,0, n}=\left(\sum_{j=1}^{q} W_{n, j}\left\|\theta_{n, j}\right\|^{2}\right)^{1 / 2}=k_{n}^{1 / 2}\left(\sum_{j=1}^{q} W_{j}\left\|\theta_{n, j}\right\|^{2}\right)^{1 / 2}$, the following inequality holds:

$$
\begin{aligned}
\frac{1}{2}\left\|\theta_{n+1}^{-}\right\|^{2}+m \int_{I_{n}}\left\|C D_{0, x}^{\beta} \theta\right\|^{2} d t+C m \int_{I_{n}}\|\theta\|^{2} d t\|\theta\|_{0,2, n} \\
\leq\left\|\theta_{n}^{-}\right\|^{2}+\sum_{i=1}^{q}\left\{\left\|R_{1}\right\|^{2}+\left\|R_{2}\right\|^{2}+\left\|c D_{0, x}^{\beta} R_{3}\right\|^{2}+\left\|C D_{0, x}^{\beta} R_{4}\right\|^{2}\right\} \\
+C \sum_{i=1}^{q}\left\|\theta_{n, i}\right\|^{2}+C h_{n}^{2 s}\|u\|_{s}^{2}+C h_{n-1}^{2 s}\|u\|_{s}^{2} .
\end{aligned}
$$

Taking the interpolation representation of $\theta$ on time slab $I_{n}$ into the error equation (4.22), and multiplying $\tau_{i}^{-1 / 2}$ in both side of the error equation, we have

$$
\begin{aligned}
& -\sum_{j=1}^{q} W_{n, j} l_{n, i}^{\prime}\left(t_{n, j}\right) \tau_{j}^{1 / 2} \tau_{i}^{-1 / 2}\left(\tilde{\theta}_{n, j}, \phi\right)+W_{n, j} \tau_{j}^{1 / 2} \tau_{i}^{-1 / 2}\left({ }_{c} D_{0, x}^{\beta} a(U) \tilde{\theta}_{n, j}, c D_{x, b}^{\beta} \phi\right) \\
& \quad+W_{n, j} \tau_{j}^{1 / 2} \tau_{i}^{-1 / 2}\left(b(U) \tilde{\theta}_{n, j}, c D_{x, b}^{\beta} \phi\right)+\delta_{q i}\left(\tilde{\theta}_{n, j}, \phi\right) \\
& =\tau_{i}^{-1 / 2}\left[\left(\theta_{n}^{-}, l_{n, i}\left(t_{n}\right) \phi\right)+\left(R_{1}, \phi\right)+\left(R_{2}, \phi\right)+\left(R_{3, c} D_{x, b}^{\beta} \phi\right)+\left(R_{4, c} D_{x, b}^{\beta} \phi\right)+\left(R_{5}, \phi\right)\right] .
\end{aligned}
$$

Let $\phi=\tilde{\theta}_{n, i}$, and sum from $i=1$ to $q$, with Lemma 3.2, we know that

$$
\sum_{i=1}^{q} \delta_{q i}\left(\tilde{\theta}_{n, q}, \tilde{\theta}_{n, i}\right)-\sum_{i, j=1}^{q} \tau_{i}^{-1 / 2} \tau_{j}^{1 / 2}\left(\tilde{\theta}_{n, j}, \tilde{\theta}_{n, i}\right)=\left(\widetilde{M} \tilde{\theta}_{n}, \tilde{\theta}_{n}\right) \geq \alpha \sum_{j=1}^{q}\left\|\tilde{\theta}_{n, j}\right\|^{2},
$$

where $\tilde{\theta}_{n}=\left(\tilde{\theta}_{n, 1}, \tilde{\theta}_{n, 2}, \ldots, \tilde{\theta}_{n, q}\right)$.

The second term of the left side of (4.42) is estimated as

$$
\begin{aligned}
\sum_{i=1}^{q} W_{n, i}\left(c D_{0, x}^{\beta} a(U) \tilde{\theta}_{n, i}, c D_{x, b}^{\beta} \tilde{\theta}_{n, i}\right) & \geq m \sum_{i=1}^{q} W_{n, j}\left(c D_{0, x}^{\beta} \tilde{\theta}_{n, i}, c D_{x, b}^{\beta} \tilde{\theta}_{n, i}\right) \\
& \geq m C \sum_{i=1}^{q}\left\|c D_{0, x}^{\beta} \tilde{\theta}_{n, i}\right\|^{2}, \\
\sum_{i=1}^{q} W_{n, i}\left(b(U) \tilde{\theta}_{n, i}, c D_{x, b}^{\beta} \tilde{\theta}_{n, i}\right) & \geq m \sum_{i=1}^{q} W_{n, i}\left(\tilde{\theta}_{n, i}, c D_{x, b}^{\beta} \tilde{\theta}_{n, i}\right) \\
& \geq m C \sum_{i=1}^{q} W_{n, i}\left\|\tilde{\theta}_{n, i}\right\| \cdot\left\|c D_{x, b}^{\beta} \tilde{\theta}_{n, i}\right\| \\
& \geq m C k_{n}^{1 / 2} \sum_{i=1}^{q}\left\|c D_{0, x}^{\beta} \tilde{\theta}_{n, i}\right\|^{2} .
\end{aligned}
$$


From (4.39)-(4.40), the following estimation can be derived:

$$
\begin{aligned}
\alpha \sum_{i=1}^{q}\left\|\tilde{\theta}_{n, i}\right\|^{2}+m c \sum_{i=1}^{q}\left\|c D_{0, x}^{\beta} \tilde{\theta}_{n, i}\right\|^{2}+m C k_{n}^{1 / 2} \sum_{i=1}^{q}\left\|C D_{0, x}^{\beta} \tilde{\theta}_{n, i}\right\|^{2} \\
\leq C\left(\sum_{i=1}^{q}\left\|\tilde{\theta}_{n, i}\right\|^{2}\right)^{1 / 2}\left\{\left\|\theta_{n}^{-}\right\|^{2}+\left(\sum _ { i = 1 } ^ { q } \left(\left\|R_{1}\right\|^{2}+\left\|R_{2}\right\|^{2}+\left\|c D_{0, x}^{\beta} R_{3}\right\|^{2}\right.\right.\right. \\
\left.\left.\left.+\left\|c D_{0, x}^{\beta} R_{4}\right\|^{2}\right)\right)^{1 / 2}+\left\|\left[u_{n}-P_{h} u_{n}\right]\right\|\right\} .
\end{aligned}
$$

Introduce the estimations from $R_{1}$ to $R_{4}$ and the inequality containing $\left\|\left[u_{n}-P_{h} u_{n}\right]\right\|$ into the above expression, by the definition and characteristics of the norm of the fractional derivative space and the relation of $\|\theta\|_{0, \beta, n}$, there holds

$$
\begin{aligned}
\|\theta\|_{0, \beta, n} \leq C k_{n} & \left\{\left\|\theta_{n}^{-}\right\|_{\beta}+h_{n}^{s}\left\|u_{t}\right\|_{0, s, n}+h_{n}^{s-\beta}\|u\|_{0, s, n}+k_{n}^{q}\left(\left\|u^{(q+1)}\right\|_{0,0, n}^{2}\right)\right. \\
& \left.+\left\|u^{(q)}\right\|_{0, s, n}+\left\|C D_{0, x}^{2 \beta} u(q)\right\|_{0,0, n}+\left\|C D_{0, x}^{\beta} u(q)\right\|_{0,0, n}+\left(h_{n}^{s}+h_{n-1}^{s}\right)\|u\|_{s}\right\} .
\end{aligned}
$$

Introducing the estimations from $R_{1}$ to $R_{5}$ into (4.37), based on the boundedness of the fractional operation, one has

$$
\begin{aligned}
\left\|\theta_{n+1}^{-}\right\|^{2} & \leq C k_{n}^{2 q}\left(\left\|u^{(q+1)}\right\|_{0,0, n}^{2}+\left\|u^{(q)}\right\|_{0,0, n}^{2}+\left\|{ }_{C} D_{0, x}^{2 \beta} u^{(q)}\right\|_{0,0, n}^{2}+\left\|c D_{0, x}^{\beta} u^{(q)}\right\|_{0,0, n}^{2}\right) \\
& +\left(h_{n}^{2 s}+h_{n-1}^{2 s}\right)\|u\|_{s}^{2}+h_{n}^{2 s}\left(\left\|u_{t}\right\|_{0, s, n}^{2}+h_{n}^{-2 s}\left\|u_{t}\right\|_{0, s, n}^{2}\right)+C\|\theta\|_{0, \beta, n}^{2}+\left\|\theta_{n}^{-}\right\|^{2} .
\end{aligned}
$$

From inequalities (4.42) and (4.43), there holds

$$
\begin{aligned}
\left\|\theta_{n+1}^{-}\right\|^{2} \leq & C k_{n}^{2 q}\left(1+k_{n}\right)\left(\left\|u^{(q+1)}\right\|_{0,0, n}^{2}+\left\|c D_{0, x}^{2 \beta} u^{(q)}\right\|_{0,0, n}^{2}+\left\|c D_{0, x}^{\beta} u^{(q)}\right\|_{0,0, n}^{2}\right) \\
& +\left(1+k_{n}\right)\left(h_{n}^{2 s}+h_{n-1}^{2 s}\right)\|u\|_{s}^{2}+\left(1+k_{n}\right) h_{n}^{2 s}\left\|u_{t}\right\|_{0, s, n}^{2}+\left(1+k_{n}\right)\left\|\theta_{n}^{-}\right\|_{\beta}^{2} .
\end{aligned}
$$


According to the above inequality, we can estimate $\theta_{n}^{-}$. With the $\theta_{j}, 0 \leq j \leq n$,

$$
\begin{aligned}
\left\|\theta_{n+1}^{-}\right\|_{\beta}^{2} \leq \prod_{j=0}^{n} C\left(1+k_{j}\right)\left\|\theta_{0}\right\|_{\beta}^{2} & \\
+C \sum_{m=0}^{n} \prod_{j=m+1}^{n}\left(1+k_{j}\right)\{C & k_{m}^{2 q}\left(\left\|u^{(q+1)}\right\|_{0,0, m}^{2}+\left\|c D_{0, x}^{2 \beta} u^{(q)}\right\|_{0,0, m}^{2}+\left\|c D_{0, x}^{\beta} u^{(q)}\right\|_{0,0, m}^{2}\right) \\
& +\left(h_{m}^{2 s}+h_{m-1}^{2 s}\right)\|u\|_{s}^{2}+h^{2 s}\left\|u_{t}\right\|_{0, s, n} \\
& \left.+\left(1+k_{n}\right) h_{n}^{2 s-2 \beta}\|u\|_{0, s, n}+k_{n}^{2 q}\left(1+k_{n}\right)\left\|\mathrm{u}^{(\mathrm{q})}\right\|_{0,0, \mathrm{n}}^{2}\right\} .
\end{aligned}
$$

For a fixed $n$, if $k_{j} \rightarrow 0, j=1,2, \ldots, n$, we choose $k=\max _{0 \leq j \leq n} k_{j}$, then there holds

$$
\prod_{j=0}^{n}\left(1+k_{j}\right) \leq \prod_{j=0}^{n}(1+k)=(1+k)^{n} \leq e^{C t_{n+1}}
$$

Setting $C_{1}=e^{C t_{n+1}}$, since $\theta_{0}^{-}=u_{0}-P_{h} u_{0}$, we get

$$
\begin{aligned}
\left\|\theta_{n+1}^{-}\right\|^{2} \leq C\left\|u_{0}-P_{h} u_{0}\right\|_{\beta}^{2}+C_{1} \sum_{m=0}^{n}\{ & k_{m}^{2 q}\left(\left\|u^{(q+1)}\right\|_{0,0, m}^{2}+\left\|{ }_{c} D_{0, x}^{2 \beta} u^{(q)}\right\|_{0,0, m}^{2}+\left\|c D_{0, x}^{\beta} u^{(q)}\right\|_{0,0, m}^{2}\right) \\
& +\left(h_{m}^{2 s}+h_{m-1}^{2 s}\right)\|u\|_{s}^{2}+h_{m}^{2 s}\left\|u_{t}\right\|_{0, s, m}+h^{2 s-2 \beta}\|u\|_{s}^{2} \\
& \left.+\left(1+k_{n}\right) h_{n}^{2 s-2 \beta}\|u\|_{0, s, n}+k_{n}^{2 q}\left(1+k_{n}\right)\left\|\mathrm{u}^{(\mathrm{q})}\right\|_{0,0, \mathrm{n}}^{2}\right\} .
\end{aligned}
$$

Taking it into (4.43), we have

$$
\begin{aligned}
\|\theta\|_{0, \beta, n} \leq C k_{n}^{1 / 2} h_{0}\left\|u_{0}\right\|_{s}+C_{1} k_{n}^{1 / 2} \sum_{m=0}^{n}\{ & k_{m}^{2 q}\left(\left\|u^{q+1}\right\|_{0,0, m}^{2}+\left\|c D_{0, x}^{2 \beta} u^{(q)}\right\|_{0,0, m}^{2}+\left\|c D_{0, x}^{\beta} u^{(q)}\right\|_{0,0, m}^{2}\right) \\
& +\left(h_{m}^{2 s}+h_{m-1}^{2 s}\right)\|u\|_{s}^{2}+h_{m}^{2 s}\left\|u_{t}\right\|_{0, s, m}+\left(1+k_{n}\right) h_{n}^{2 s-2 \beta}\|u\|_{0, s, n} \\
& \left.+k_{n}^{2 q}\left(1+k_{n}\right)\left\|u^{(q)}\right\|_{0,0, n}^{2}\right\} .
\end{aligned}
$$

Note that $\|U-u\|_{\infty, \beta, n} \leq\|\theta\|_{\infty, \beta, n}+\|W-u\|_{\infty, \beta, n}$. By Lemma 2.8, the estimate of $\|W-u\|$ is proved. Therefore, the proof of Theorem 4.5 is ended. 
Table 1: Numerical error result for Example 5.1.

\begin{tabular}{ccc}
\hline$h$ & $\left\|u-u_{h}\right\|_{\infty, 0}$ & Cvge. rate \\
\hline $1 / 10$ & $0.1220 e-003$ & - \\
$1 / 20$ & $0.7433 e-004$ & 0.7147 \\
$1 / 40$ & $0.3244 e-004$ & 1.1962 \\
$1 / 80$ & $0.1642 e-004$ & 0.9828 \\
$1 / 160$ & $0.7880 e-005$ & 1.0588 \\
$1 / 320$ & $0.3952 e-005$ & 0.9955 \\
\hline
\end{tabular}

\section{Numerical Examples}

In this section, we present numerical results for the Galerkin approximations which support the theoretical analysis derived in Section 4.

Let $S_{h}$ denote a uniform partition on spacial domain $[0, a]$ and $X_{h}$ the space of continuous piecewise linear functions on $S_{h}$. In order to implement the discontinuous Galerkin finite element approximation, we adapt the finite elements scheme in space domain and the discontinuous finite element scheme along time domain. We associate shape function of space $X_{h}$ with the standard basis of hat functions on the uniform grid of size $h=1 / n$ and adapt the same shape functions along time axis. In our scheme, the finite element trial and test spaces for Problem 1 are chosen to be the same.

Example 5.1. Consider the following equation:

$$
\begin{gathered}
\frac{\partial u}{\partial t}=\left(-{ }_{c} D_{0, x}^{1.6} \frac{x^{1.6}}{\Gamma(4.6)}-{ }_{c} D_{0, x}^{0.8} \frac{x^{0.8}}{\Gamma(3.8)}\right) u, \quad 0 \leq x \leq 1,0 \leq t \leq 1, \\
u(x, 0)=x^{2}, \quad 0 \leq x \leq 1, \\
u(0, t)=0, \quad u(1, t)=e^{t}, \quad 0 \leq t \leq 1 .
\end{gathered}
$$

The exact solution of the equation is $u(x, t)=e^{-t} x^{2}$.

If we select $k_{n}=c h$ and note $u(x, 0)$ is smooth enough, then we have the following numerical results presented in Table 1 .

Table 1 includes numerical calculations over a regular partition of $[0,1]$. We can see that, when the size of grid becomes smaller, the finite element approximation becomes better. We can also observe that the experimental rates of convergence agree well with the theoretical rates for the numerical solution.

Example 5.2. We consider another space fractional nonlinear Fokker-Planck differential equation. The $u(x, t)=e^{-t} x^{3}$ is the exact solution of the following equation:

$$
\begin{gathered}
\frac{\partial u}{\partial t}=\left(-{ }_{c} D_{0, x}^{1.8} \frac{12 e^{t} u}{\Gamma(5.8) x^{1.2}}+{ }_{c} D_{0, x}^{0.9} \frac{6 e^{t} u}{\Gamma(4.9) x^{2.1}}\right) u(x, t), \quad x \in(0,2), t \in[0,1), \\
u(x, 0)=x^{3}, \quad 0 \leq x \leq 2, \\
u(0, t)=0, \quad u(1, t)=e^{t}, \quad 0 \leq t \leq 1 .
\end{gathered}
$$


Table 2: Numerical error result for Example 5.2.

\begin{tabular}{ccc}
\hline$h$ & $\left\|u-u_{h}\right\|_{\infty, 0}$ & Cvge. rate \\
\hline $1 / 10$ & 0.0231 & - \\
$1 / 20$ & 0.0127 & 0.8610 \\
$1 / 40$ & 0.0067 & 0.9315 \\
$1 / 80$ & 0.0034 & 0.9660 \\
$1 / 160$ & 0.0017 & 0.9830 \\
$1 / 320$ & 0.0009 & 0.9915 \\
\hline
\end{tabular}

Table 3: Numerical error result for Example 5.3.

\begin{tabular}{ccc}
\hline$h$ & $\left\|u-u_{h}\right\|_{\infty, 0}$ & Cvge. rate \\
\hline $1 / 10$ & $0.6125 e-003$ & - \\
$1 / 20$ & $0.4199 e-003$ & 0.5447 \\
$1 / 40$ & $0.1765 e-003$ & 1.2500 \\
$1 / 80$ & $0.0985 e-003$ & 0.8425 \\
$1 / 160$ & $0.0527 e-003$ & 0.9003 \\
\hline
\end{tabular}

We can also select $k_{n}=c h$. Table 2 shows the error results at different sizes of space grid. We can still observe that the experimental rates of convergence are inline with the theoretical convergence rates.

Example 5.3. Equation $u(x, t)=e^{-t}\left(x^{2}-x^{1.9}\right)$ is the exact solution of the following problem:

$$
\begin{gathered}
\frac{\partial u}{\partial t}=-{ }_{c} D_{0, x}^{1.6} \frac{\left(x^{-0.4} u+x^{1.5}\right) u}{\Gamma(4.6)}+\frac{x^{0.9} \Gamma(3.5)}{\Gamma(4.6) \Gamma(1.9)}-\frac{x^{2}}{2} e^{-t}, \quad 0 \leq x \leq 1,0 \leq t \leq 1, \\
u(x, 0)=x^{2}-x^{1.9}, \quad 0 \leq x \leq 1, \\
u(0, t)=0, \quad u(1, t)=0, \quad 0 \leq t \leq 1 .
\end{gathered}
$$

Table 3 includes numerical calculations over a regular partition of $[0,1]$. We can see that the experimental numerical is valid and inline with the theoretical result. We can also see that this method is valid for more generalized fractional diffusion-type equation, and the rate of convergence depends on the highest order of the equation and the order of the shape function.

\section{Acknowledgments}

This work was partially supported by the National Natural Science Foundation of China under Grant no. 10872119, Shanghai Leading Academic Discipline Project under Grant no. S30104, and the Natural Science Foundation of Anhui province Grant no. KJ2010B442. 


\section{References}

[1] P. Hänggi and H. Thomas, "Stochastic processes: time evolution, symmetries and linear response," Physics Reports, vol. 88, no. 4, pp. 207-319, 1982.

[2] H. Sun, W. Chen, C. Li, and Y. Chen, "Fractional differential models for anomalous diffusion," Physica A, vol. 389, no. 14, pp. 2719-2724, 2010.

[3] R. Metzler, E. Barkai, and J. Klafter, "Deriving fractional Fokker-Planck equations from a generalised master equation," Europhysics Letters, vol. 46, no. 4, pp. 431-436, 1999.

[4] A. Dubkov and B. Spagnolo, "Generalized Wiener process and Kolmogorov's equation for diffusion induced by non-Gaussian noise source," Fluctuation and Noise Letters, vol. 5, no. 2, pp. L267-L274, 2005.

[5] R. Metzler and J. Klafter, “The random walk's guide to anomalous diffusion: a fractional dynamics approach," Physics Reports, vol. 339, no. 1, p. 77, 2000.

[6] A. A. Dubkov, B. Spagnolo, and V. V. Uchaikin, "Lévy flight superdiffusion: an introduction," International Journal of Bifurcation and Chaos, vol. 18, no. 9, pp. 2649-2672, 2008.

[7] C. Tsallis and E. K. Lenzi, "Anomalous diffusion: nonlinear fractional Fokker-Planck equation," Chemical Physics, vol. 284, no. 1-2, pp. 341-347, 2002.

[8] S. I. Denisov, W. Horsthemke, and P. Hänggi, "Generalized Fokker-Planck equation: derivation and exact solutions," The European Physical Journal B, vol. 68, no. 4, pp. 567-575, 2009.

[9] G. Jumarie, "Fractional Brownian motions via random walk in the complex plane and via fractional derivative. Comparison and further results on their Fokker-Planck equations," Chaos, Solitons and Fractals, vol. 22, no. 4, pp. 907-925, 2004.

[10] A. A. Dubkov, A. L. Cognata, and B. Spagnolo, "The problem of analytical calculation of barrier crossing characteristics for Lévy flights," Journal of Statistical Mechanics: Theory and Experiment, vol. P01002, no. 1, pp. 1-12, 2009.

[11] D. Brockmann and I. M. Sokolov, "Lévy flights in external force fields: from models to equations," Chemical Physics, vol. 284, no. 1-2, pp. 409-421, 2002.

[12] Z. Odibat and S. Momani, "Numerical solution of Fokker-Planck equation with space- and timefractional derivatives," Physics Letters A, vol. 369, no. 5-6, pp. 349-358, 2007.

[13] V. E. Tarasov and G. M. Zaslavsky, "Fokker-Planck equation with fractional coordinate derivatives," Physica A, vol. 387, no. 26, pp. 6505-6512, 2008.

[14] C. Li and Y. Wang, "Numerical algorithm based on Adomian decomposition for fractional differential equations," Computers \& Mathematics with Applications, vol. 57, no. 10, pp. 1672-1681, 2009.

[15] Y. Zheng, C. Li, and Z. Zhao, "A note on the finite element method for the space-fractional advection diffusion equation," Computers \& Mathematics with Applications, vol. 59, no. 5, pp. 1718-1726, 2010.

[16] F. Liu, V. Anh, and I. Turner, "Numerical solution of the space fractional Fokker-Planck equation," Journal of Computational and Applied Mathematics, vol. 166, no. 1, pp. 209-219, 2004.

[17] B. Baeumer, M. Kovács, and M. M. Meerschaert, "Numerical solutions for fractional reaction-diffusion equations," Computers \& Mathematics with Applications, vol. 55, no. 10, pp. 2212-2226, 2008.

[18] C. Li and C. Tao, "On the fractional Adams method," Computers \& Mathematics with Applications, vol. 58, no. 8, pp. 1573-1588, 2009.

[19] G. J. Fix and J. P. Roop, "Least squares finite-element solution of a fractional order two-point boundary value problem," Computers E Mathematics with Applications, vol. 48, no. 7-8, pp. 1017-1033, 2004.

[20] V. J. Ervin and J. P. Roop, "Variational formulation for the stationary fractional advection dispersion equation," Numerical Methods for Partial Differential Equations, vol. 22, no. 3, pp. 558-576, 2006.

[21] J. P. Roop, "Computational aspects of FEM approximation of fractional advection dispersion equations on bounded domains in $R^{2}$, " Journal of Computational and Applied Mathematics, vol. 193, no. 1, pp. 243-268, 2006.

[22] H. Brunner and D. Schötzau, "Hp-discontinuous Galerkin time-stepping for Volterra integrodifferential equations," SIAM Journal on Numerical Analysis, vol. 44, no. 1, pp. 224-245, 2006.

[23] A. Lasis and E. Süli, "Hp-version discontinuous Galerkin finite element method for semilinear parabolic problems," SIAM Journal on Numerical Analysis, vol. 45, no. 4, pp. 1544-1569, 2007.

[24] H. Li and R. X. Liu, "The space-time finite element method for parabolic problems," Applied Mathematics and Mechanics, vol. 22, no. 6, pp. 613-624, 2001. 
[25] T. Sun and K. Ma, "A space-time discontinuous Galerkin method for linear convection-dominated Sobolev equations," Applied Mathematics and Computation, vol. 210, no. 2, pp. 490-503, 2009.

[26] J. Alberty and C. Carstensen, "Discontinuous Galerkin time discretization in elastoplasticity: motivation, numerical algorithms, and applications," Computer Methods in Applied Mechanics and Engineering, vol. 191, no. 43, pp. 4949-4968, 2002.

[27] K. Wang, H. Wang, S. Sun, and M. F. Wheeler, "An optimal-order $\mathrm{L}_{2}$-error estimate for nonsymmetric discontinuous Galerkin methods for a parabolic equation in multiple space dimensions," Computer Methods in Applied Mechanics and Engineering, vol. 198, no. 27-29, pp. 2190-2197, 2009. 


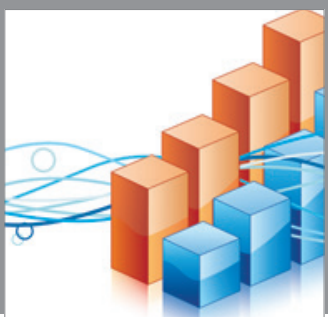

Advances in

Operations Research

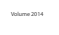

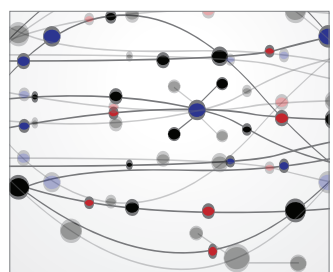

\section{The Scientific} World Journal
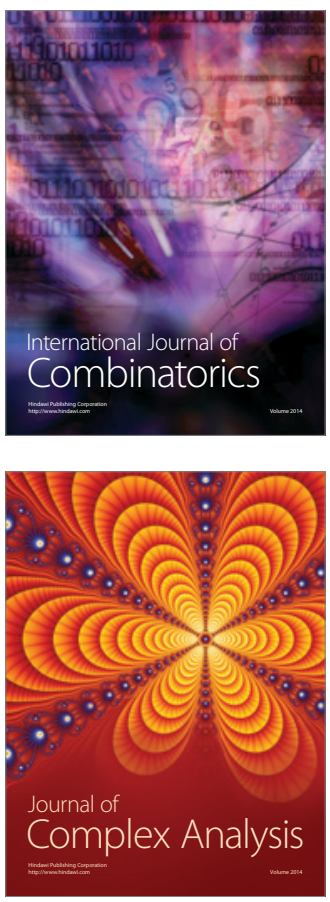

International Journal of

Mathematics and

Mathematical

Sciences
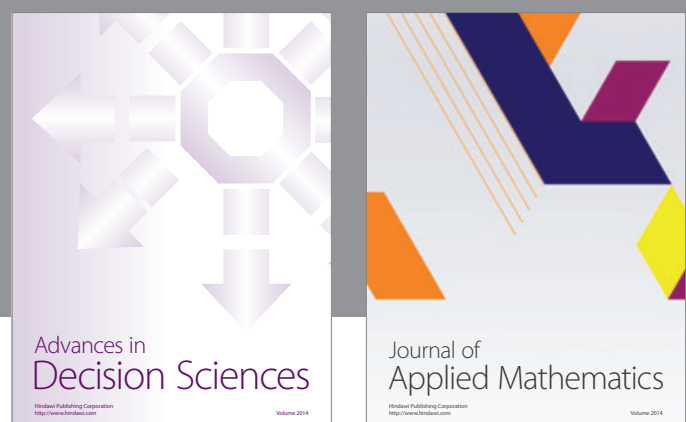

Journal of

Applied Mathematics
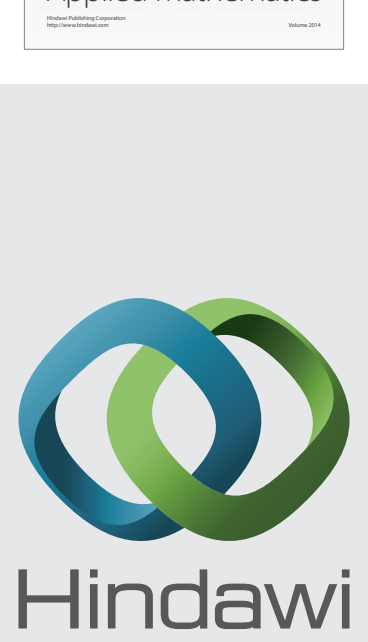

Submit your manuscripts at http://www.hindawi.com
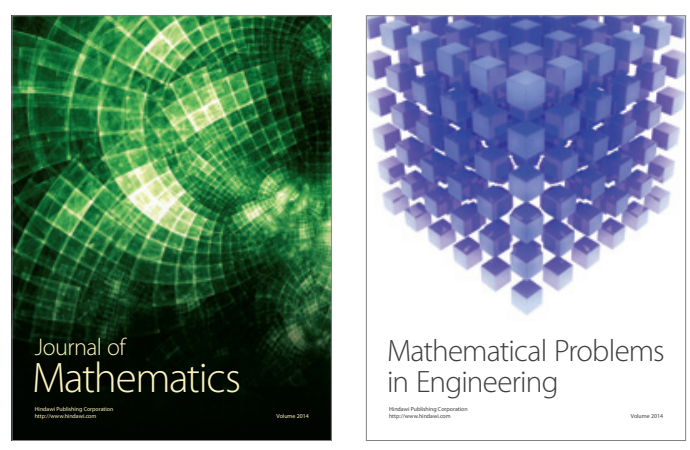

Mathematical Problems in Engineering
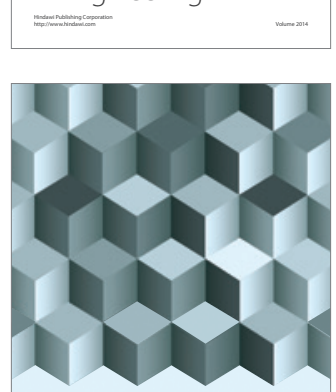

Journal of

Function Spaces
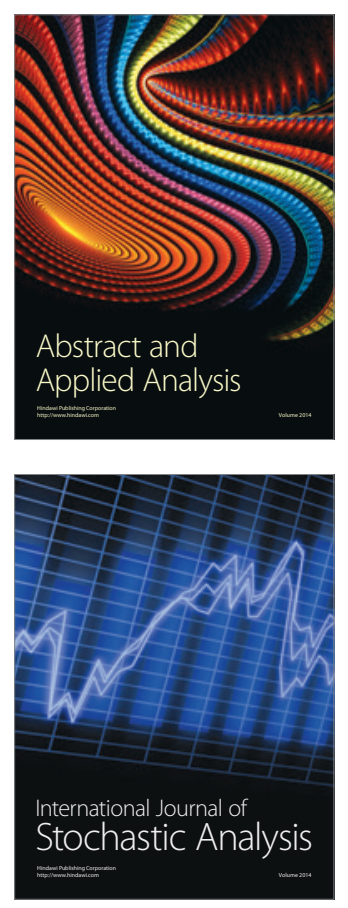

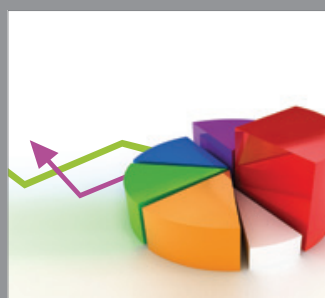

ournal of

Probability and Statistics

Promensencen
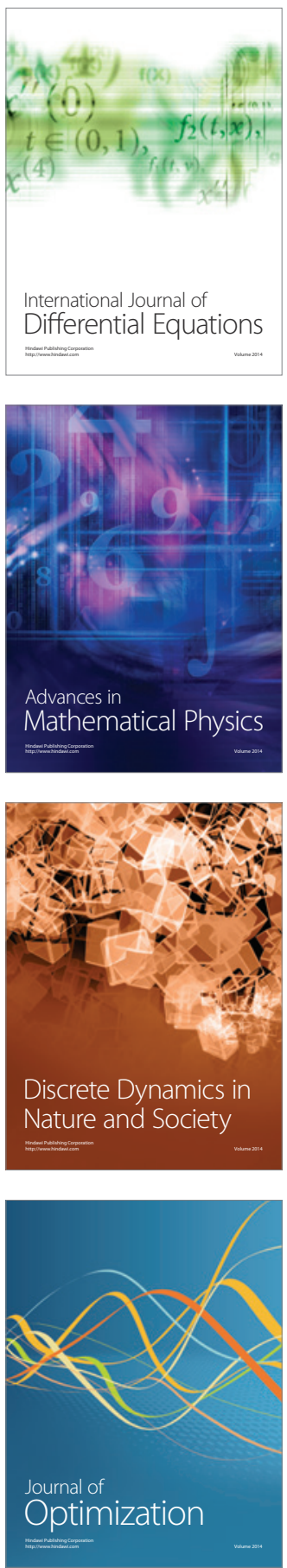